\title{
Symbolic Analysis Based on Graph Transformations
}

\author{
Zdenek Kolka, Martin Vlk, Dalibor Biolek, Viera Biolkova \\ Faculty of Electrical Engineering and Communication \\ Brno University of Technology \\ Brno, Czech Republic \\ kolka@feec.vutbr.cz
}

\begin{abstract}
The paper deals with implementation details of a method for approximate symbolic analysis of linear circuits based on nontrivial transformations of voltage and current graphs. The method is based on eliminating the low-voltage branches from "high-voltage" loops and the low-current branches from "high-current" cuts. This goes beyond the simple edge deletion or contraction used in previous methods. The paper describes a graph transformation for decreasing the number of spanning trees and its computer implementation.
\end{abstract}

\section{INTRODUCTION}

An arbitrary network function in the frequency domain of lumped, linear and time-invariant circuits can be expressed symbolically as

$$
F(s, \mathbf{p})=\frac{s^{m} q_{m}(\mathbf{p})+\ldots+q_{0}(\mathbf{p})}{s^{n} r_{n}(\mathbf{p})+\ldots+r_{0}(\mathbf{p})}
$$

where $\mathbf{p}=\left(p_{1}, p_{2}, \ldots, p_{k}\right)^{T}$ is a vector of the parameters of network elements (network parameters), $s$ is the complex frequency, and $q_{i}(\mathbf{p}), \quad r_{i}(\mathbf{p})$ are the sum-of-product expressions of network parameters. The complexity of (1) grows exponentially with circuit size [1].

It has been found that the majority of symbolic terms can be removed from large expressions without any significant numerical error [2]. This is the basic principle of all symbolic simplification methods, which are based on a combination of symbolic and numerical analyses. Usually, the approximated expression validity is checked numerically at several points $(f, \mathbf{p})_{i}$ in the frequency-parameter space.

The method described here belongs to the Simplification Before Generation (SBG) class where graphs or matrices representing the circuit are modified before the symbolic analysis in order to decrease the number of symbolic terms. A method, published as Sifting Approach [1], is based on a heuristic algorithm consisting in eliminating the device parameters from the numerator and the denominator submatrices separately. Another method [2], called Twograph Simplification, deletes or contracts the edges of voltage and current graphs constructed for the numerator and the denominator separately.
We have proposed a different approach based on the twograph method. Instead of simply deleting or contracting the graph edges it modifies the graph structure in order to decrease the number of common spanning trees. Graphtheoretical proofs were published in [3]. This paper deals with an improved version exploiting the block decomposition of graphs.

\section{Motivating EXAMPLE}

Let us consider a simple circuit in Fig. 1, whose network parameters are: $R_{B}=36 \mathrm{k} \Omega, r_{\pi}=4 \mathrm{k} \Omega, g_{m}=35 \mathrm{mS}, R_{L}=4 \mathrm{k} \Omega$.

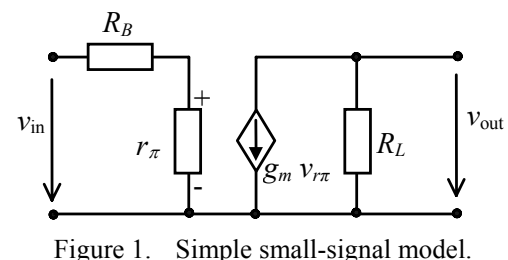

The exact formula for the voltage transfer ratio is

$$
K_{V}=\frac{v_{\text {out }}}{v_{\text {in }}}=-\frac{r_{\pi}}{R_{B}+r_{\pi}} g_{m} R_{L}
$$

As $R_{B}>>r_{\pi}$, the formula can be further simplified. Let us limit ourselves to the SBG methods only. Resistor $r_{\pi}$ cannot be simply removed or its terminal shorted. Both operations would cause an unacceptable error.

The circuit can be solved by the two-graph method [4]. The determinant of the nodal admittance matrix is

$$
\operatorname{det} \mathbf{Y}=\sum_{t \in T\left(G_{\mathrm{V}}\right) \cap T\left(G_{\mathrm{I}}\right)} \varepsilon(t) Y^{(t)},
$$

where $Y^{(t)}$ is the tree-admittance product of tree $t, T\left(G_{\mathrm{V}}\right)$ and $T\left(G_{\mathrm{I}}\right)$ are the sets of all spanning trees of voltage and current graphs. The intersection $T\left(G_{\mathrm{V}}\right) \cap T\left(G_{\mathrm{I}}\right)$ represents the common spanning trees of $G_{\mathrm{I}}$ and $G_{\mathrm{V}} . \varepsilon(t)= \pm 1$ is the tree sign. The technique of augmented circuit [2] allows computing both the numerator and the denominator at the same time. Fig. 2 shows an example for voltage transfer.

Research described in the paper was financially supported by the Czech Grant Agency under grants No. 102/05/0771 and No. 102/05/0277, and by the Czech Ministry of Education under research program MSM0021630513. 


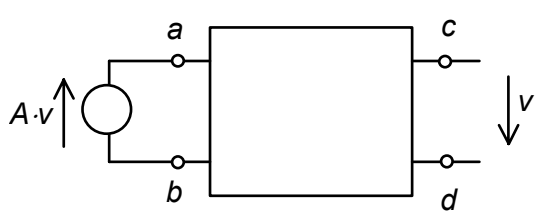

Figure 2. Augmented circuit for voltage transfer ratio.

The VCVS in Fig. 2 is represented by a suitable admittance model [5]. The determinant of the admittance matrix is

$$
\begin{gathered}
\Delta=\Delta_{1}+A \Delta_{2}, \text { and } \\
K_{V}=\frac{\Delta_{2}}{\Delta_{1}} .
\end{gathered}
$$

Since $A$ is a symbol, the terms belonging to $\Delta_{1}$ or $\Delta_{2}$ are easy to recognize.

Fig. 3a shows voltage and current graphs for the augmented circuit. Edges " 1 " and " $A$ " model the VCVS [5].

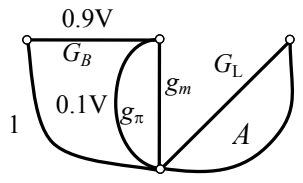

a)

voltage graph

b)

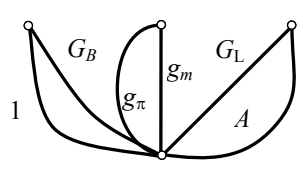

Figure 3. a) Original and b) modified graphs.

Let the voltage across input edge " 1 " be $1 \mathrm{~V}$. Then the voltage across $r_{\pi}$ is $0.1 \mathrm{~V}$. The voltage can be neglected in loops $1-G_{B^{-}} g_{\pi}$ and $1-G_{B^{-}} g_{m}$ but not in loop $g_{\pi}-g_{m}$. A simple modification in Fig. $3 b$ removes $g_{m}$ and $g_{\pi}$ from the "highvoltage" loops only. The simplified formula for the voltage transfer ratio obtained using (4) and (5) is then

$$
K_{V}^{\prime}=-\frac{r_{\pi}}{R_{B}} g_{m} R_{L}
$$

An inspection of the voltage and current graphs from Fig. $3 \mathrm{~b}$ shows that they represent a CCCS instead of $r_{\pi}$ and VCCS.

This simple example shows the basic principle of the proposed method - selective removal of the low-voltage edges from the "high-voltage" loops. Similar transformations can be found for cuts of the current graph. The method will be called topological simplification (TSBG).

\section{TOPOLOGICAL SBG}

\section{A. Graph Transformations}

Let $G$ be a graph. Then $V(G)$ is a set of its vertices, $E(G)$ is a set of its edges, and $T(G)$ is a set of its trees. The incidence of edge $e$ in graph $G, \rho(e, G)=(i, j)$, assigns two vertices $i, j$ to edge $e$. An edge with the incidence $(v, v)$ is called selfloop. Graph $G$ is said to be separable if there is a vertex whose removal splits the graph into two or more components. A block is a maximal nonseparable subgraph.

The basic operation of TSBG is the separation of a connected subgraph [3].

Definition 1: Separation of a connected subgraph $G_{\mathrm{S}}$ from a graph $G$ is an operation that transforms $G$ into

$$
G^{\prime}=\widetilde{G} \cup G_{\mathrm{S}},
$$

where $\widetilde{G}$ is a subgraph whose edge set is $E(\widetilde{G})=E\left(G \backslash G_{\mathrm{S}}\right)$. The incidence $\rho(e, G)=\left(v_{i}, v_{j}\right)$ of any edge $e \in E(\widetilde{G})$ is transformed into $\rho(e, \widetilde{G})=\left(f\left(v_{i}\right), f\left(v_{j}\right)\right)$, where $f$ is

$$
f(v)=\left\langle\begin{array}{ll}
v_{\mathrm{c}} & \text { if } v \in V\left(G_{\mathrm{S}}\right) \\
v & \text { otherwise }
\end{array} .\right.
$$

$v_{\mathrm{c}}$ is an arbitrary but fixed vertex $v_{\mathrm{c}} \in V\left(G \backslash G_{\mathrm{S}}\right) \cap V\left(G_{\mathrm{S}}\right)$. The operations will be denoted as follows:

$$
\widetilde{G}=G \Rightarrow G_{\mathrm{S}}, \quad G^{\prime}=G \triangleright G_{\mathrm{S}} .
$$
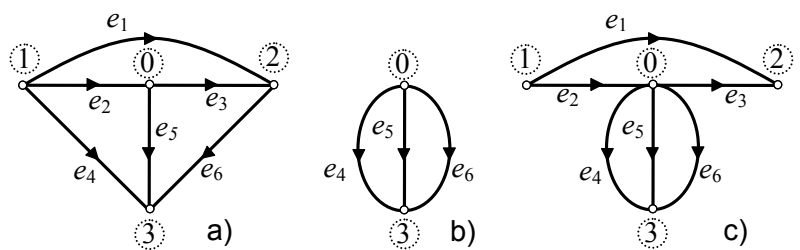

Figure 4. Example of separation $G_{\mathrm{S}}=\left\{e_{1}, e_{2}, e_{3}\right\}$ : a) original graph $G$; b) graph $\widetilde{G}=G \Rightarrow G_{\mathrm{S}}$; c) $G^{\prime}=G \triangleright G_{S}$.

Fig. 4 demonstrates the separation of $G_{\mathrm{S}}=\left\{e_{1}, e_{2}, e_{3}\right\}$. The transformation does not change the number of edges and vertices and decreases the number of spanning trees of $G$. Proof can be found in [3].

\section{B. Loop and Cut Transformations}

Let the circuit be represented by the current graph $G_{\mathrm{I}}$ and the voltage graph $G_{\mathrm{V}}$ with edges $e_{1}, e_{2}, \ldots, e_{b}$, whose weights are the magnitudes of branch currents and voltages for a particular frequency.

Let $L_{1}, L_{2}, \ldots, L_{B} \subseteq G_{\mathrm{V}}$ be all the loops of the voltage graph $G_{\mathrm{V}}$. The voltage $v\left(e_{j}\right)$ of an edge $e_{j} \in E\left(L_{i}\right)$ will be considered numerically negligible in loop $L_{i}$ if

$$
\left|v\left(e_{j}\right)\right|<\varepsilon_{\mathrm{V}} \max _{h \in E\left(L_{i}\right)}|v(h)|,
$$

where $\varepsilon_{\mathrm{V}} \in(0,1)$ is the threshold value. Such an edge is a candidate for being removed from $L_{i}$.

Let us assume that the voltage graph $G_{\mathrm{V}}$ can be decomposed into two edge-disjoint subgraphs $G_{\mathrm{V}}^{\mathrm{H}}$ and $G_{\mathrm{V}}^{\mathrm{L}}$ and the condition 


$$
\max _{e \in G_{\mathrm{V}}^{\mathrm{L}} \cap L}|v(e)|<\varepsilon_{\mathrm{V}} \max _{e \in L}|v(e)|,
$$

holds for any loop $L \subseteq G_{\mathrm{V}}$ that is contained in both subgraphs. Then it is possible to remove the low-voltage edges of $G_{\mathrm{V}}^{\mathrm{L}}$ from high-voltage loops by the separation

$$
G_{\mathrm{V}}^{\prime}=G_{\mathrm{V}} \triangleright G_{\mathrm{V}}^{\mathrm{L}}
$$

In the example from Section 2, $G_{\mathrm{V}}^{\mathrm{L}}=\left\{g_{\pi}, g_{m}\right\}$. The separation of a disconnected subgraph is performed component-by-component.
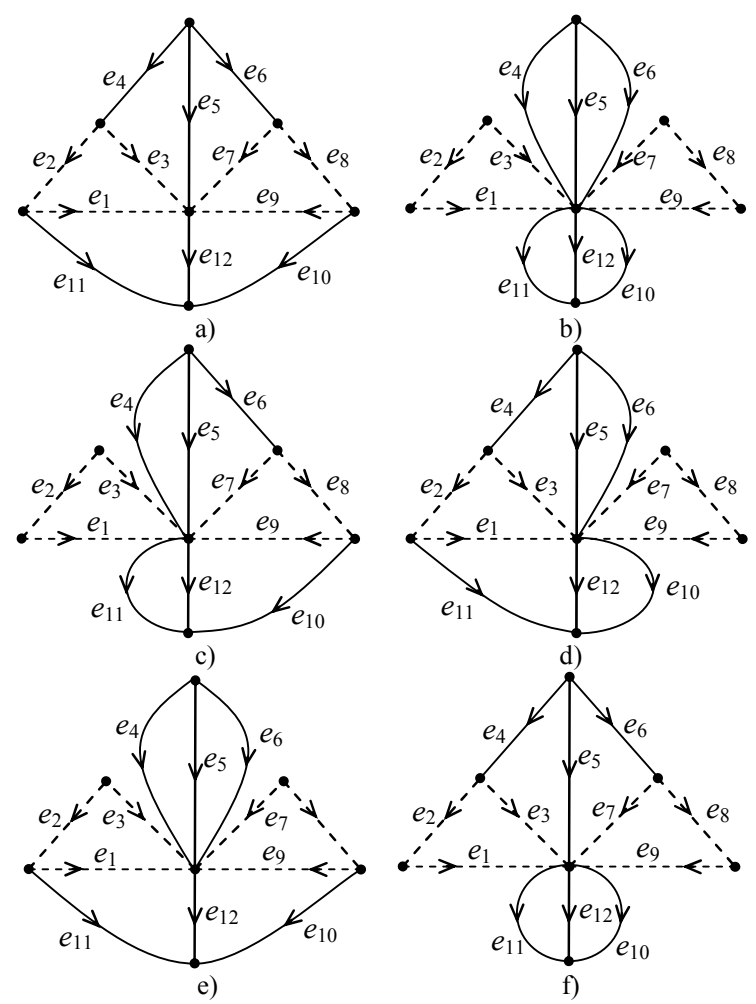

Figure 5. Fine-grained separations: a) original graph,

b) $G \triangleright G_{S}$, c) $G \triangleright\left\{e_{1}, e_{2}, e_{3}\right\}$, d) $G \triangleright\left\{e_{7}, e_{8}, e_{9}\right\}$

e) $\left.G \triangleright\left(G_{S} \cup\left\{e_{10}, e_{11}, e_{12}\right\}\right), \mathrm{f}\right) G \triangleright\left(G_{S} \cup\left\{e_{4}, e_{5}, e_{6}\right\}\right)$.

Subgraph separation (12) can be divided into elementary operations exploiting the block structure of both $G_{\mathrm{V}}^{\mathrm{L}}$ and $\widetilde{G}_{V}$. Condition (10) has been formulated for loops contained in both subgraphs. Let us assume that $G_{\mathrm{V}}^{\mathrm{L}}$ is separable with blocks $B_{i}^{\mathrm{V}}$. As there is no loop contained in two or more blocks, the separation of any block $B_{i}^{\mathrm{V}}$, which is considered the elementary operation, satisfies (10) as well. Graph $\widetilde{G}_{V}=G_{V} \Rightarrow G_{V}^{L}$ may also be separable. The loops contained in $\widetilde{G}_{V}$ form disjoint subsets. It is easy to augment $G_{\mathrm{V}}^{\mathrm{L}}$ such that the resulting graph $\widetilde{G}_{V}^{\prime}$ becomes nonseparable.
Fig. $5 \mathrm{~b}$ shows the separation of subgraph $G_{S}=\left\{e_{1}, e_{2}, e_{3}, e_{7}, e_{8}, e_{9}\right\}$ having two blocks $\left(B_{1}=\left\{e_{1}, e_{2}, e_{3}\right\}\right.$ and $\left.B_{2}=\left\{e_{7}, e_{8}, e_{9}\right\}\right)$ that can be separated independently, Fig. 5c,d. $\widetilde{G}$ consists of two blocks $\left(\widetilde{B}_{1}=\left\{e_{4}, e_{5}, e_{6}\right\}\right.$, $\left.\widetilde{B}_{2}=\left\{e_{10}, e_{11}, e_{12}\right\}\right)$. Augmenting $G_{S}$ by the complement of $\widetilde{B}_{1}$ or $\widetilde{B}_{2}$ results in nonseparable $\widetilde{G}^{\prime}$, Fig. 5e,f.

It can be also shown that removing small-current branches from high-current cuts leads to decomposing $G_{\mathrm{I}}$ with respect to a threshold value $\varepsilon_{I} \in(0,1)$ into two edgedisjoint subgraphs $G_{\mathrm{I}}^{\mathrm{H}}$ and $G_{\mathrm{I}}^{\mathrm{L}}$ [3]. If for any loop $L \subseteq G_{\mathrm{I}}$ contained in both subgraphs the condition

$$
\min _{e \in E(L)}|i(e)|<\varepsilon_{\mathrm{I}} \min _{e \in E\left(G_{\mathrm{I}}^{\mathrm{H}} \cap L\right)}|i(e)|
$$

holds then it is possible to perform the separation

$$
G_{\mathrm{I}}^{\prime}=G_{\mathrm{I}} \triangleright G_{\mathrm{I}}^{\mathrm{H}} \text {. }
$$

\section{Relation to classical methods}

Let us consider the separation of an edge $e_{\mathrm{c}}$ with admittance parameter $y_{\mathrm{c}}$ from a graph $G$, Fig. $6 \mathrm{~b}$.

$$
G^{\prime}=G \triangleright\left\{e_{\mathrm{c}}\right\} .
$$

All the spanning trees of $G^{\prime}$ contain the edge $e_{\mathrm{c}}$. Thus all numerator and denominator terms contain $y_{\mathrm{c}}$, which can be evidently reduced. The separation is equivalent to $y_{\mathrm{c}} \rightarrow \infty$.
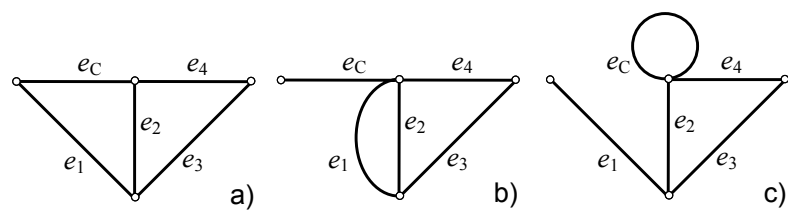

Figure 6. a) Graph $G$; b) $G^{\prime}=G \triangleright\left\{e_{\mathrm{c}}\right\}$; c) $G^{\prime \prime}=G \triangleright\left\{\bar{e}_{\mathrm{c}}\right\}$.

The separation of complement $\overline{e_{\mathrm{c}}}=G \backslash e_{\mathrm{c}}$ of edge $e_{\mathrm{c}}$

$$
G^{\prime \prime}=G \triangleright\left\{\overline{e_{c}}\right\}
$$

always forms a self-loop in $G$ ', No tree can contain $y_{\mathrm{c}}$. The separation is equivalent to $y_{\mathrm{c}} \rightarrow 0$. Thus the methods of [1] and [2] are just special cases of a more general TSBG.

\section{ALGORITHMS}

The error-control strategy is similar to the method of [6]. Assuming $m$ reference points the error criterion is

$$
\varepsilon_{A}=\max _{i=1 . . m} \frac{20 \log \left|E\left(\omega_{i}\right)\right|}{\Delta M_{\omega_{i}}}+\frac{\arg \left(E\left(\omega_{i}\right)\right)}{\Delta \varphi_{\omega_{i}}},
$$

where $E(\omega)=F_{A}(\omega) / F_{R}(\omega)$ and $\Delta M_{\omega_{i}}, \Delta \varphi_{\omega_{i}}$ are inverse weights. $F_{A}(\omega)$ and $F_{R}(\omega)$ are approximated and reference network functions, respectively. 


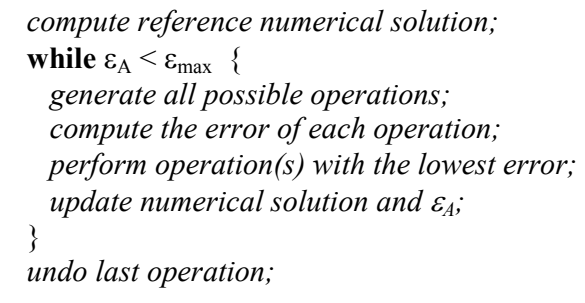

Figure 7. Main cycle of simplification method.

The simplification algorithm is applied twice. First, it performs the parametric simplification, i.e. the elementary operations are $p_{i} \rightarrow 0$ and $p_{i} \rightarrow \infty$ for each parameter. The method of large-change sensitivity [7] is used for error computation. This step substantially decreases the circuit size. The cost is approximately $O\left(r m n^{3}\right)$, where $n$ is the circuit matrix order, $r$ is the number of removed parameters, and $m$ is the number of reference points.

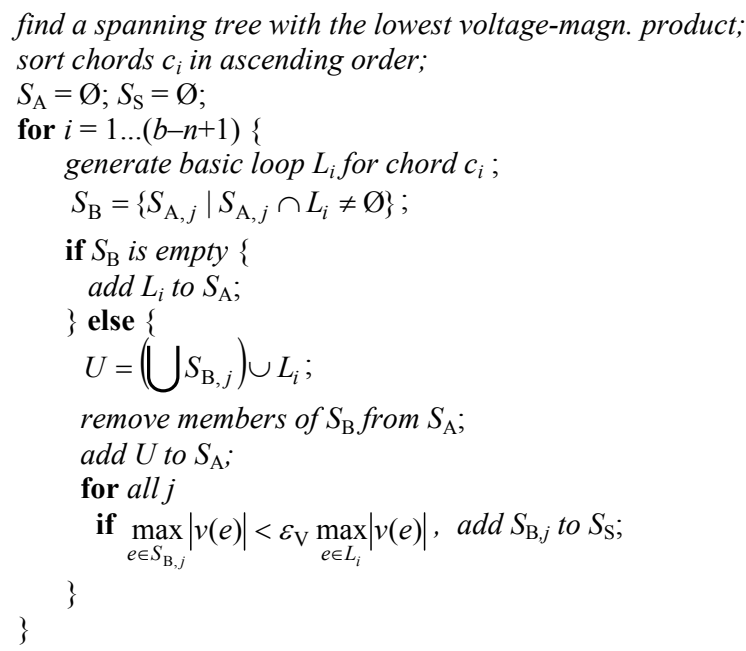

Figure 8. Generation of elementary operations for $G_{\mathrm{V}}$.

The next step consists in separating the subgraphs (or their blocks) that fulfill (11) or (13), Fig. 8. Let the graph $\left(G_{\mathrm{V}}\right.$ or $\left.G_{\mathrm{I}}\right)$ be connected and have $n$ vertices and $b$ edges. Any spanning tree $t$ consists of exactly $n-1$ edges $-t w i g s$. The remaining edges will be called chords [7]. A basic loop is formed from one chord and some twigs. Let $S_{\mathrm{A}}$ and $S_{\mathrm{B}}$ be auxiliary sets of subgraphs and $S_{\mathrm{S}}$ a set of candidates for separation. The symbol $S_{\mathrm{A}, i}$ represents the $i$-th member of $S_{\mathrm{A}}$. The algorithm for the current graph is similar. Candidates are generated for all frequency samples. The cost is approximately $O\left(m\left(n^{3}+b\right)\right)$.

\section{EXAMPLE ANALYSIS}

The method is demonstrated on an analysis of a currentfeedback amplifier in Matlab. Parametric preprocessing reduced the original 100 network parameters to 14 . The topological algorithm separated two subgraphs from the voltage graph and one subgraph from the current graph. The formula for voltage transfer was further simplified by means of the SAG method [6].

TABLE I. RESULTS OF SIMPLIFICATION STEPS

\begin{tabular}{|l|l|l|l|l|}
\hline \multicolumn{2}{|c|}{ accuracy } & \multicolumn{1}{c|}{ parametric } & \multicolumn{1}{c|}{ graph-based } & \multicolumn{1}{c|}{ SAG } \\
\hline$\Delta M_{\max }$ & allowed & \multicolumn{2}{|c|}{ $\pm 1 \mathrm{~dB} @ 1 \mathrm{kHz}$ and $600 \mathrm{kHz}$} \\
\hline & actual & $0.47 \mathrm{~dB} @ 1 \mathrm{kHz}$ & $0.56 \mathrm{~dB} @ 600 \mathrm{kH}$ & $0.21 \mathrm{~dB} @ 1 \mathrm{kHz}$ \\
\hline$\Delta \varphi_{\max }$ & allowed & \multicolumn{3}{|c|}{ $\pm 3^{\circ} @ 1 \mathrm{kHz}$ and $600 \mathrm{kHz}$} \\
\hline & actual & $2.4^{\circ} @ 600 \mathrm{kHz}$ & $2.4^{\circ} @ 600 \mathrm{kHz}$ & $3.0^{\circ} @ 600 \mathrm{kHz}$ \\
\hline \# of numer. terms & 44 & 4 & 3 \\
\hline \# of denom. terms & 232 & 65 & 46 \\
\hline \# of parameters & 14 & 14 & 14 \\
\hline runtime & $4.9 \mathrm{~s}$ & $1.3 \mathrm{~s}$ & $0.9 \mathrm{~s}$ \\
\hline
\end{tabular}

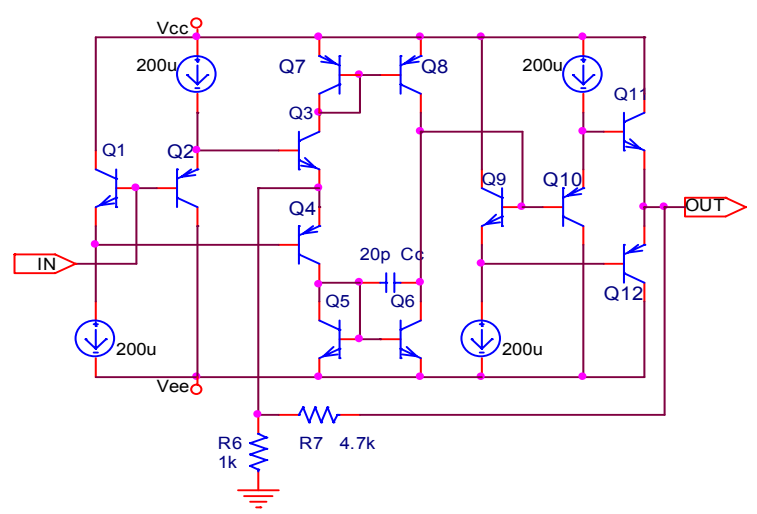

Figure 9. Current-feedback amplifier.

\section{CONCLUSION}

The method of topological simplification allows reaching a higher degree of simplification than the parameter-based SBG techniques do. The result is a simplified circuit model that is analyzed symbolically. An important property is that the graph transformation is based on physical relations in the circuit in contrast to the rather mathematical methods.

\section{REFERENCES}

[1] J.J. Hsu and C. Sechen, "DC small signal symbolic analysis of large analog integrated circuits," IEEE Trans. Circuits Syst-I, vol. 41, no. 12, pp.817-828, 1994.

[2] Q. Yu and C. Sechen, "A Unified Approach to the Approximate Symbolic Analysis of Large Analog Integrated Circuits," IEEE Trans. Circuits Syst-I, vol. 43, no. 8, pp.656-669, 1996.

[3] Z. Kolka, M. Vlk, D. Biolek, V. Biolkova, "Topology Transformations for Symbolic Analysis", Proc. of the 2006 IEEE Int'1 Midwest Symposium on Circuits and Systems, Puerto Rico, USA, 2006, pp. $200-204$.

[4] W. Mayeda and S. Seshu, Topological Formulas for Network Functions, Eng. Exp. Station, University of Illinois, Bulletin 446, Urbana, 1957.

[5] M. Vlk, Z. Kolka, "New Topological Approach to Simplification Before Generation", Proc. of 7th Int'l Workshop on Symb. Methods and Applications to Circuit Design, Sinaia, 2002, pp. 120 - 123.

[6] R. Sommer, E. Hennig, and G. Dröge, "Equation-Based Symbolic Approximation by Matrix Reduction with Quantitative Error Prediction," Alta-Frequenza, vol.5, no.6, pp.29-37, 1993.

[7] J. Vlach and K. Singhal, Computer Methods for Circuit Analysis and Design, 2nd edition, Van Nostrad Reinhold, 1994. 